\title{
Adição Simultânea de Sulfato de Amônio e Ureia à Calda de PUlverizaÇÃo do Herbicida GlyPhosate ${ }^{1}$
}

\author{
Simultaneous Addition of Ammonium Sulfate and Urea to Glyphosate Spray Solution \\ CARVALHO, S.J.P. ${ }^{2}$, DIAS, A.C.R. ${ }^{3}$, SHIOMI, G.M. ${ }^{4}$ e CHRISTOFFOLETI, P.J. ${ }^{5}$
}

\begin{abstract}
RESUMO - Dois experimentos foram desenvolvidos em casa de vegetação, com o objetivo de avaliar a eficácia do herbicida glyphosate sobre plantas de Digitaria insularis quando soluções de ureia (U; $\left.5 \mathrm{~g} \mathrm{~L}^{-1}\right)$, sulfato de amônio (SA; $\left.15 \mathrm{~g} \mathrm{~L}^{-1}\right)$ ou U+SA $\left(2,5+7,5 \mathrm{~g} \mathrm{~L}^{-1}\right)$ foram utilizadas como veículo de pulverização. Aos 28 dias após aplicação, de acordo com as curvas de doseresposta (primeiro experimento), foram necessários $409 \mathrm{~g} \mathrm{ha}^{-1}$ de glyphosate para atingir $50 \%$ de controle da planta daninha $\left(\mathrm{C}_{50}\right)$ quando água sem adjuvantes foi usada como veículo de pulverização. Para obtenção dos mesmos 50\% de controle, as doses de glyphosate foram reduzidas para 373, 208 e $189 \mathrm{~g} \mathrm{ha}^{-1}$, quando o herbicida foi pulverizado utilizando solução de $\mathrm{U}, \mathrm{SA}$ ou U+SA, respectivamente. A redução na dose oriunda da combinação de glyphosate e U+SA também foi observada para controles de $80 \%\left(\mathrm{C}_{80}\right)$. No segundo experimento, a adição de U+SA à calda elevou o controle obtido com a menor dose de glyphosate (360 $\left.\mathrm{g} \mathrm{ha}^{-1}\right)$, igualando-o à aplicação da maior dose $\left(720 \mathrm{~g} \mathrm{ha}^{-1}\right)$ sem adjuvantes. Esses resultados evidenciam efeito complementar de U e SA em elevar a eficácia do glyphosate para controle de $D$. insularis.
\end{abstract}

Palavras-chave: Digitaria insularis, curva dose-resposta, dessecação, nitrogênio.

\begin{abstract}
Two trials were carried out under greenhouse conditions to evaluate the efficacy of glyphosate on Digitaria insularis plants when urea (U; $5 \mathrm{~g} \mathrm{~L}^{-1}$ ); ammonium sulfate (AMS; $15 \mathrm{~g} \mathrm{~L} \mathrm{~L}^{-1}$ ) or U+AMS $\left(2.5+7.5 \mathrm{~g} \mathrm{~L}^{-1}\right)$ were used as spray solutions. At 28 days after application, according to dose-response curves (first trial), $409 \mathrm{~g} \mathrm{ha}^{-1}$ of glyphosate application were necessary to obtain 50\% of weed control $\left(C_{50}\right)$ when water without adjuvants was used as spray solution. To reach the same $50 \%$ of weed control, glyphosate rates were reduced to 373, 208 and $189 \mathrm{~g} \mathrm{ha}^{-1}$, when the herbicide was sprayed using a solution of $U$, AMS or $U+A M S$, respectively. Reduction in the dose of glyphosate combined with $U+A M S$ was also observed for controls of $80 \%\left(C_{80}\right)$. In the second trial, the addition of U+SA to glyphosate spray solution increased the control obtained at the lowest glyphosate rate (360 $\left.\mathrm{g} \mathrm{ha}^{-1}\right)$, leveling it to the highest rate application (720 $\mathrm{g} \mathrm{ha}^{-1}$ ) without adjuvants. These results show the complementary action of U and AMS to increase glyphosate efficacy to control $\boldsymbol{D}$. insularis.
\end{abstract}

Keywords: Digitaria insularis, dose-response curve, desiccation, nitrogen.

\section{INTRODUÇÃO}

A eficácia dos herbicidas aplicados às folhas das plantas daninhas está diretamente relacionada com a magnitude dos processos de penetração/absorção (Durigan, 1992).
Esses processos são complexos e podem ser influenciados por fatores relacionados à planta, aos herbicidas, à calda de pulverização e ao clima (Durigan, 1992; Silva et al., 2007). Segundo Monquero et al. (2004), a absorção foliar do glyphosate é um processo bifásico, que

1 Recebido para publicação em 31.9.2009 e na forma revisada em 3.9.2010.

Parte da tese do primeiro autor apresentada à Escola Superior de Agricultura "Luiz de Queiroz" - Esalq/USP para obtenção do título de Doutor em Ciências/Fitotecnia.

2 Professor do Instituto Federal do Sul de Minas, Campus Machado, Caixa Postal 1004, CEP 37750-971 Machado-MG, <sjpcarvalho@yahoo.com.br>; ${ }^{3}$ Doutoranda em Fitotecnia, Esalq/USP, <anacarolina.r.dias@gmail.com>; ${ }^{4}$ Eng ${ }^{-}-$Agr ${ }^{0}$., <g.shiomi@yahoo.com.br>; ${ }^{5}$ Professor Associado do Dep. de Produção Vegetal, ESALQ/USP, <pjchrist@esalq.usp.br>. 
envolve a rápida penetração pela cutícula, seguida de lenta absorção simplástica. Durante essas etapas, a cutícula foliar e a membrana plasmática têm sido consideradas barreiras importantes que limitam a atividade do herbicida (Satchivi et al., 2000).

O glyphosate [N-(fosfonometil)glicina] é herbicida não-seletivo, de ação sistêmica, usado no controle de plantas daninhas anuais e perenes e na dessecação das culturas de cobertura (Rodrigues \& Almeida, 2005). A absorção do glyphosate ocorre pelas estruturas fotossinteticamente ativas das plantas e, dentro das células, suas moléculas inibem a EPSPs (enol piruvil shiquimato fosfato sintase), enzima que participa da rota de sintese dos aminoácidos aromáticos fenilalanina, tirosina e triptofano (Geiger \& Fuchs, 2002).

Visando facilitar a penetração foliar de xenobióticos, diversas substâncias podem ser adicionadas à calda de pulverização. Nesse sentido, a ureia tem sido utilizada em aplicações foliares como fonte de nitrogênio e, principalmente, como agente facilitador da penetração de nutrientes catiônicos, aniônicos e herbicidas (Yamada et al., 1965; Freire et al., 1981; Durigan, 1992). A adição de sulfato de amônio à calda de pulverização também tem sido associada à maior eficácia de herbicidas (Nalewaja \& Matysiak, 1993; Pratt et al., 2003; Young et al., 2003; Carvalho et al., 2008, 2009a). Contudo, não foram encontrados trabalhos que avaliaram a adição concomitante de ureia e sulfato de amônio à calda de pulverização.

Das plantas daninhas comumente encontradas em áreas agrícolas brasileiras, o capimamargoso (Digitaria insularis) tem ganhado especial atenção, sobretudo por exigir aplicação de doses de glyphosate superiores às recomendadas para adequado controle de outras espécies da família Poaceae. Segundo Timossi et al. (2006), a aplicação de $1.440 \mathrm{~g} \mathrm{ha}^{-1} \mathrm{de}$ glyphosate promoveu controle satisfatório da comunidade infestante, porém não evitou o rebrote do capim-amargoso. A maior dificuldade em controlar plantas de capim-amargoso originadas de rizomas pode estar relacionada com a maior espessura na epiderme das faces adaxial e abaxial e maior espessura da lâmina foliar, quando comparadas com plantas provenientes de sementes (Machado et al., 2008).
Ainda, esses autores observaram grande quantidade de amido nos rizomas, o que pode dificultar a translocação do glyphosate e permitir rápido rebrote da parte aérea das plantas.

Nesse sentido, dois experimentos foram desenvolvidos em casa de vegetação com o objetivo de avaliar a eficácia do herbicida glyphosate sobre plantas de capim-amargoso quando pulverizado com solução de sulfato de amônio (SA), ureia (U) ou U+SA.

\section{MATERIAL E MÉTODOS}

Dois experimentos foram desenvolvidos em casa de vegetação do Departamento de Produção Vegetal da Escola Superior de Agricultura "Luiz de Queiroz" - Esalq/USP, no município de Piracicaba (22 $42^{\circ}$ ' $30^{\prime \prime}$ de latitude sul, 47 ${ }^{\circ} 38^{\prime} 00^{\prime \prime}$ de longitude oeste e $546 \mathrm{~m}$ de altitude), Estado de São Paulo, entre julho e dezembro de 2008. Sementes de capim-amargoso (Digitaria insularis), considerada como planta-teste, foram coletadas em área experimental da Esalq/USP com histórico de aplicações ocasionais de glyphosate. Os propágulos foram acondicionados em sacos de papel, em local seco, à temperatura ambiente, até a instalação dos experimentos.

Inicialmente, as sementes de capimamargoso germinaram em caixas plásticas com capacidade para $2 \mathrm{~L}$, preenchidas com substrato comercial (casca de Pinus + turfa + vermiculita). Em estádio fenológico de uma folha plenamente expandida, as plântulas foram transplantadas para vasos, onde permaneceram até o final do experimento, em população média de oito plantas por vaso. Em ambos os experimentos, adotou-se delineamento experimental de blocos ao acaso, em que cada parcela constou de um vaso com capacidade para 1,5 L, preenchido com mistura de substrato comercial e vermiculita, na proporção de $2: 1$, respectivamente. Todas as parcelas foram fertilizadas com (mg por parcela): 280 de $\mathrm{N}, 280$ de $\mathrm{P}_{2} \mathrm{O}_{5}, 280$ de $\mathrm{K}_{2} \mathrm{O}, 4$ de $\mathrm{B}, 6$ de $\mathrm{Cu}$, 4 de Fe, 4 de Mg, 0,2 de Mo e 4 de Zn. Os vasos foram mantidos em casa de vegetação com irrigação automatizada por aspersão.

No primeiro experimento, os tratamentos foram organizados segundo esquema fatorial, com sete doses do herbicida glyphosate (Roundup Original $^{\circledR}$ ): 5.760; 1.440; 360; 90; 22,5 
ou 5,6 g e.a. ha ${ }^{-1}$, ou ausência de herbicida; e quatro veículos de aplicação: solução de ureia (U; $5 \mathrm{~g} \mathrm{~L}^{-1}$ ), solução de sulfato de amônio (SA; $\left.15 \mathrm{~g} \mathrm{~L}^{-1}\right)$, solução de U+SA $\left(2,5+7,5 \mathrm{~g} \mathrm{~L}^{-1}\right)$ ou água pura. As doses utilizadas nos fertilizantes nitrogenados foram fundamentadas em observações prévias, relatadas por Carvalho et al. (2008, 2009a,b). Os fertilizantes nitrogenados foram provenientes de fontes convencionalmente utilizadas nas adubações agrícolas, com concentrações de 21 e $45 \%$ de nitrogênio no sulfato de amônio e ureia, respectivamente.

A água utilizada em todas as aplicações, inclusive para o preparo das soluções, foi oriunda de tratamento comum para distribuição pública (Tabela 1), sendo classificada quanto à dureza como água branda (Silva et al., 2007). Ainda, amostras dos quatro veículos de aplicação (soluções preparadas) foram encaminhadas ao Laboratório de Isótopos Estáveis, do Centro de Energia Nuclear na Agricultura - CENA, onde foram mensurados o $\mathrm{pH}$ e os teores de amônio $\left(\mathrm{NH}_{4}^{+}\right)$. $\mathrm{O} \mathrm{pH}$ foi mensurado por meio do medidor digital de $\mathrm{pH}$, modelo TEC-3MP. Para determinação do amônio, as amostras foram destiladas com $\mathrm{MgO}$ e os extratos titulados com ácido sulfúrico, segundo recomendação de Bremmer \& Keeney (1966).
O segundo experimento teve por objetivo comprovar os resultados obtidos com o primeiro trabalho. Para elevar a precisão experimental, foram utilizados seis tratamentos com as doses que promoveram a maior amplitude de resposta no primeiro experimento (análise gráfica visual) e seis repetições. Os tratamentos utilizados no segundo experimento estão descritos na Tabela 2 .

Todas as pulverizações foram realizadas em câmara de aplicação fechada, pressurizada por ar comprimido, utilizando-se uma ponta do tipo leque (Teejet 80.02), distanciada em $0,50 \mathrm{~m}$ da superficie do alvo e com volume relativo de calda equivalente a $200 \mathrm{~L} \mathrm{ha}^{-1}$. No momento das aplicações, constatou-se estádio fenológico de 4-5 folhas e 1-2 perfilhos para o capim-amargoso. Após cada aplicação, os vasos foram mantidos em área sem irrigação por período de 24 horas, o que assegurou a adequada absorção foliar do glyphosate.

Em ambos os experimentos, realizaramse avaliações de controle das plantas daninhas aos 14, 21 e 28 dias após aplicação (DAA) e de massa fresca e seca aos 28 DAA. Quanto às avaliações de controle, utilizou-se escala com variação entre 0 e $100 \%$, em que 0 representou a ausência de sintomas e 100 a morte de todas as plantas. Os dados de massa foram obtidos

Tabela 1 - Análise da água utilizada nas aplicações, submetida a tratamento usual para distribuição pública, realizado na Esalq/USP. Piracicaba-SP, 2008

\begin{tabular}{|c|c|c|c|c|c|}
\hline Parâmetro & Unidade & Resultado & Parâmetro & Unidade & Resultado \\
\hline Cloreto $\left(\mathrm{Cl}^{-}\right)$ & $m g L^{-1}$ & 43,6 & Zinco $(\mathrm{Zn})$ & $\mathrm{mg} \mathrm{L}^{-1}$ & 0,04 \\
\hline Nitrato $\left(\mathrm{N}-\mathrm{NO}_{3}^{-}\right)$ & $\mathrm{mg} \mathrm{L}^{-1}$ & 4,0 & Alcalinidade $\left(2 \mathrm{CO}_{3}{ }^{2-}+\mathrm{HCO}_{3}^{-}\right)$ & $\mathrm{mg} \mathrm{L}^{-1}$ & 49,6 \\
\hline Sulfato $\left(\mathrm{SO}_{4}{ }^{2-}\right)$ & $\mathrm{mg} \mathrm{L}^{-1}$ & 33,0 & Nitrogênio Amoniacal $\left(\mathrm{N}-\mathrm{NH}_{3}\right)$ & $\mathrm{mg} \mathrm{L}^{-1}$ & 0,2 \\
\hline Fósforo $(\mathrm{P})$ & $\mathrm{mg} \mathrm{L}^{-1}$ & 0,02 & Cor Aparente & $\mathrm{PtCo}$ & 0,0 \\
\hline Sódio $\left(\mathrm{Na}^{+}\right)$ & $\mathrm{mg} \mathrm{L}^{-1}$ & 66,0 & Turbidez & FTU & 0,0 \\
\hline Potássio $\left(\mathrm{K}^{+}\right)$ & $\mathrm{mg} \mathrm{L}^{-1}$ & 5,6 & Sedimentos em suspensão & $\mathrm{mg} \mathrm{L}^{-1}$ & 4,3 \\
\hline Cálcio $\left(\mathrm{Ca}^{2+}\right)$ & $\mathrm{mg} \mathrm{L}^{-1}$ & 31,8 & Condutividade elétrica (CE) & $\mathrm{mS} \mathrm{cm}^{-1}$ & 0,33 \\
\hline Magnésio $\left(\mathrm{Mg}^{2+}\right)$ & $\mathrm{mg} \mathrm{L}^{-1}$ & 2,8 & $\mathrm{pH}$ & -- & 7,5 \\
\hline Ferro $(\mathrm{Fe})$ & $\mathrm{mg} \mathrm{L}^{-1}$ & 0,0 & Gás Carbônico $\left(\mathrm{CO}_{2}\right)$ & $\mathrm{mg} \mathrm{L}^{-1}$ & 2,5 \\
\hline Cobre $(\mathrm{Cu})$ & $\mathrm{mg} \mathrm{L}^{-1}$ & 0,0 & Acidez $\left(\mathrm{CaCO}_{3}\right)$ & $\mathrm{mg} \mathrm{L}^{-1}$ & 5,0 \\
\hline Manganês (Mn) & $\mathrm{mg} \mathrm{L}^{-1}$ & 0,01 & Dureza Total $*\left(\mathrm{CaCO}_{3}\right)$ & $\mathrm{mg} \mathrm{L}^{-1}$ & 91,0 \\
\hline
\end{tabular}

Análises realizadas pelo Laboratório de Ecologia Aplicada, Departamento de Ciências Florestais, Esalq/USP. * Dureza total calculada com base no equivalente de carbonato de cálcio $\left(\mathrm{CaCO}_{3}\right)$, segundo Franson (1995): 2,497 [Ca, mg L$\left.{ }^{-1}\right]+4,118\left[\mathrm{Mg}, \mathrm{mg} \mathrm{L}^{-1}\right]$. 
Tabela 2 - Tratamentos herbicidas aplicados no segundo experimento. Piracicaba-SP, 2008

\begin{tabular}{|c|c|c|c|c|c|}
\hline \multirow{2}{*}{$N^{\text {No }}$} & \multicolumn{2}{|c|}{ Herbicida } & \multicolumn{2}{c|}{ Fertilizante nitrogenado } \\
\cline { 2 - 6 } & \multirow{2}{*}{ Condição } & \multirow{2}{*}{ Dose $\left(\mathrm{g} \mathrm{ha}^{-1}\right)$} & Tipo & \multicolumn{2}{c|}{ Dose $^{2}$} \\
\hline 1 & Testemunha sem aplicação & Ausente & 0,0 & 0,0 \\
\hline 2 & Glyphosate $^{1 /}$ & 360 & Ausente & 0,0 & 0,0 \\
\hline 3 & Glyphosate & 360 & Sulfato de Amônio & 15,0 & 3,0 \\
\hline 4 & Glyphosate & 360 & Ureia & 5,0 & 1,0 \\
\hline 5 & Glyphosate & 360 & Ureia + Sulfato de Amônio & $2,5+7,5$ & $0,5+1,5$ \\
\hline 6 & Glyphosate & 720 & Ausente & 0,0 & 0,0 \\
\hline
\end{tabular}

${ }^{1 /}$ Roundup Original ${ }^{\circledR}, 360 \mathrm{~g} \mathrm{~L}^{-1}$ de equivalente ácido; ${ }^{2 /}$ Volume de calda proporcional a $200 \mathrm{~L} \mathrm{ha}^{-1}$.

cortando-se as plantas rente à superfície do substrato. Posteriormente, o material foi seco em estufa com circulação forçada de ar, a $70{ }^{\circ} \mathrm{C}$, por 72 horas.

Todos os dados foram submetidos à aplicação do teste $\mathrm{F}$ na análise da variância. Em seguida, para o primeiro experimento, aplicouse o teste de Tukey para comparação dos níveis de tratamentos qualitativos e aplicaram-se modelos de regressão não lineares para ajuste dos niveis quantitativos. No segundo experimento, o teste de Scott-Knott (Scott \& Knott, 1974) foi utilizado para agrupamento das médias dos tratamentos. Todos os testes estatísticos foram desenvolvidos aceitando-se o nível de significância de $5 \%$.

Nas análises por regressões, a variável controle percentual foi ajustada à equação logística adaptada de Streibig (1988):

$$
y=\frac{100}{1+\left(\frac{x}{C_{50}}\right)^{\alpha}}
$$

em que $C_{50}$ é a dose de glyphosate que resulta em $50 \%$ de controle da planta daninha e $\alpha$ é a declividade da curva ao redor de $C_{50}$. Esse modelo pressupõe que, sempre que a dose de glyphosate tende ao infinito (doses elevadas), o controle tende à estabilização em 100\%.

Para as variáveis massa fresca e seca residual, adotou-se o modelo proposto por Seefeldt et al. (1995):

$$
y=a+\frac{b}{\left[1+\left(\frac{x}{c}\right)^{d}\right]}
$$

em que $y$ é a massa residual; $x$ é a dose do herbicida; e $a, b, c$ e $d$ são parâmetros do modelo, de modo que $a$ é o limite inferior da curva, $b$ é a diferença entre os pontos máximo e mínimo da curva, $c$ é a dose que proporciona $50 \%$ de resposta da variável e $d$ é a declividade da curva.

As equações logísticas apresentam vantagens, uma vez que os termos integrantes da equação são estimativas do valor de $C_{50}$ ou de $G R_{50}$ (Christoffoleti, 2002). $\mathrm{O} \mathrm{C}_{50}$ (control by $50 \%$ ) e o $G R_{50}$ (growth reduction by $50 \%$ ) são as doses do herbicida que proporcionam $50 \%$ de controle ou de redução de massa da planta daninha, respectivamente (Christoffoleti, 2002; Christoffoleti \& López-Ovejero, 2004). A fim de complementar as análises, quando necessário, também foram calculados $C_{80}$ e $G R_{80}$, utilizando-se o princípio da equação inversa, conforme discutido por Carvalho et al. (2005).

\section{RESULTADOS E DISCUSSÃO}

No primeiro experimento, foi observada interação dos veículos de aplicação com as doses de glyphosate em todas as avaliações de controle (Tabela 3; Figura 1), porém não houve interação ou mesmo efeito principal dos veículos para a avaliação de massa (Figura 2). 
A análise conjunta da Tabela 3 e da Figura 1 evidencia o controle diferencial do capimamargoso devido aos veículos de aplicação. Em todas as datas de aplicação, menores valores de $C_{50}$ ou $C_{80}$ foram observados para a pulverização de glyphosate com solução de U+SA. Em relação ao uso de água pura para pulverização do herbicida, a solução com somente sulfato de amônio também reduziu a dose de glyphosate necessária para controle de $50 \%$ da população, porém isso não foi observado para $C_{80}$ aos 14 e 21 DAA. A aplicação de glyphosate com solução de ureia não resultou em melhor controle da planta daninha (Tabela 3; Figura 1).

O herbicida glyphosate reduziu a massa fresca e seca do capim-amargoso (Figura 2), sendo as variáveis adequadamente ajustadas ao modelo logístico proposto por Seefeldt et al. (1995). Carvalho et al. (2008, 2009a) constataram melhor controle de plantas daninhas após aplicação de glyphosate com sulfato de amônio, também sem expressão diferencial dos tratamentos sobre a massa seca residual. Vale ressaltar que, nesses experimentos, o capim-amargoso correspondeu a $15 \%$ da comunidade infestante de uma das áreas experimentais.

Na literatura, diversos trabalhos comprovam a maior eficácia do glyphosate quando aplicado com sulfato de amônio. Por exemplo,
Donald (1988) concluiu que a adição de sulfato de amônio elevou a eficácia e consistência do glyphosate no controle de Hordeum jubatum; Nalewaja \& Matsiak (1993) elevaram o controle de Triticum aestivum (planta-teste) utilizando sulfato de amônio como antagonista de sais prejudiciais ao glyphosate; Jordan et al. (1997) observaram melhor controle de Sida spinosa e Ipomoea hederacea var. intergriuscula; e Pratt et al. (2003) avaliaram diversos adjuvantes para controle de Abutilon theophrasti e concluíram que nenhum foi superior ao sulfato de amônio. Embora haja disponibilidade de trabalhos que avaliaram a eficácia de glyphosate aplicado com sulfato de amônio, não foram encontrados relatos da aplicação conjunta de herbicidas com U+SA.

Com os resultados obtidos no primeiro experimento, sobretudo quanto à análise das curvas de controle aos 21 e 28 DAA (Figura 1), selecionou-se a dose de $360 \mathrm{~g} \mathrm{ha}^{-1}$ de glyphosate (maior amplitude de resposta entre os tratamentos) para validação dos dados experimentais por meio de um segundo experimento, cujos resultados estão em concordância com a discussão anterior. A aplicação de $720 \mathrm{~g} \mathrm{ha}^{-1}$ de glyphosate proporcionou os maiores percentuais de controle, sem diferença para a aplicação de $360 \mathrm{~g} \mathrm{ha}^{-1}$ de glyphosate $+\mathrm{U}+\mathrm{SA}$. Aos 21 e 28 DAA, resultados intermediários

Tabela 3 - Parâmetros do modelo logístico ${ }^{1 /}$, coeficiente de determinação $\left(\mathrm{R}^{2}\right)$ e $\mathrm{C}_{80}$ para controle do capim-amargoso (D. insularis) quando submetido a diferentes doses do herbicida glyphosate, utilizando como veículo água pura, solução de sulfato de amônio (SA; $\left.15 \mathrm{~g} \mathrm{~L}^{-1}\right)$, solução de ureia $\left(\mathrm{U} ; 5 \mathrm{~g} \mathrm{~L}^{-1}\right)$ e solução de U+SA $\left(2,5+7,5 \mathrm{~g} \mathrm{~L}^{-1}\right)$. Piracicaba-SP, 2008

\begin{tabular}{|c|c|c|c|c|c|}
\hline \multirow{2}{*}{ Variável } & \multirow{2}{*}{ Solução } & \multicolumn{2}{|c|}{ Parâmetros $^{1 /}$} & \multirow{2}{*}{$\mathrm{R}^{2}$} & \multirow{2}{*}{$\mathrm{C}_{80}$} \\
\hline & & $\mathrm{C}_{50}$ & $\alpha$ & & \\
\hline \multirow{4}{*}{$\begin{array}{l}\text { Controle } \\
14 \mathrm{DAA}^{2 /}\end{array}$} & Água & 659,691 & $-1,768$ & 0,994 & $1.445,015$ \\
\hline & Sulfato de Amônio (SA) & 523,218 & $-0,992$ & 0,998 & $2.116,401$ \\
\hline & Ureia & 613,006 & $-1,588$ & 0,994 & $1.467,559$ \\
\hline & Ureia + SA & 364,042 & $-1,185$ & 0,997 & $1.172,787$ \\
\hline \multirow{4}{*}{$\begin{array}{l}\text { Controle } \\
21 \text { DAA }\end{array}$} & Água & 529,448 & $-2,446$ & 0,991 & 933,177 \\
\hline & Sulfato de Amônio (SA) & 315,914 & $-1,176$ & 0,997 & $1.026,892$ \\
\hline & Ureia & 410,978 & $-2,012$ & 0,997 & 818,565 \\
\hline & Ureia + SA & 226,095 & $-1,529$ & 0,999 & 559,824 \\
\hline \multirow{4}{*}{$\begin{array}{l}\text { Controle } \\
28 \text { DAA }\end{array}$} & Água & 409,341 & $-3,792$ & 0,997 & 590,006 \\
\hline & Sulfato de Amônio (SA) & 207,828 & $-1,631$ & 0,999 & 486,228 \\
\hline & Ureia & 373,406 & $-2,435$ & 0,996 & 659,833 \\
\hline & Ureia + SA & 189,404 & $-1,713$ & 0,998 & 425,456 \\
\hline
\end{tabular}

1/ Modelo: $\mathrm{y}=100 /\left(1+\left(\mathrm{x} / \mathrm{C}_{50}\right)^{\alpha}\right) ;{ }^{2 /} \mathrm{DAA}-$ dias após aplicação. 
foram obtidos com a aplicação da menor dose de glyphosate em combinação com SA; já a aplicação isolada de glyphosate $\left(360 \mathrm{~g} \mathrm{ha}^{-1}\right)$ ou em combinação com $U$ promoveu controles mais baixos (Tabela 4).

Com relação à massa fresca residual do capim-amargoso, observou-se diferenciação
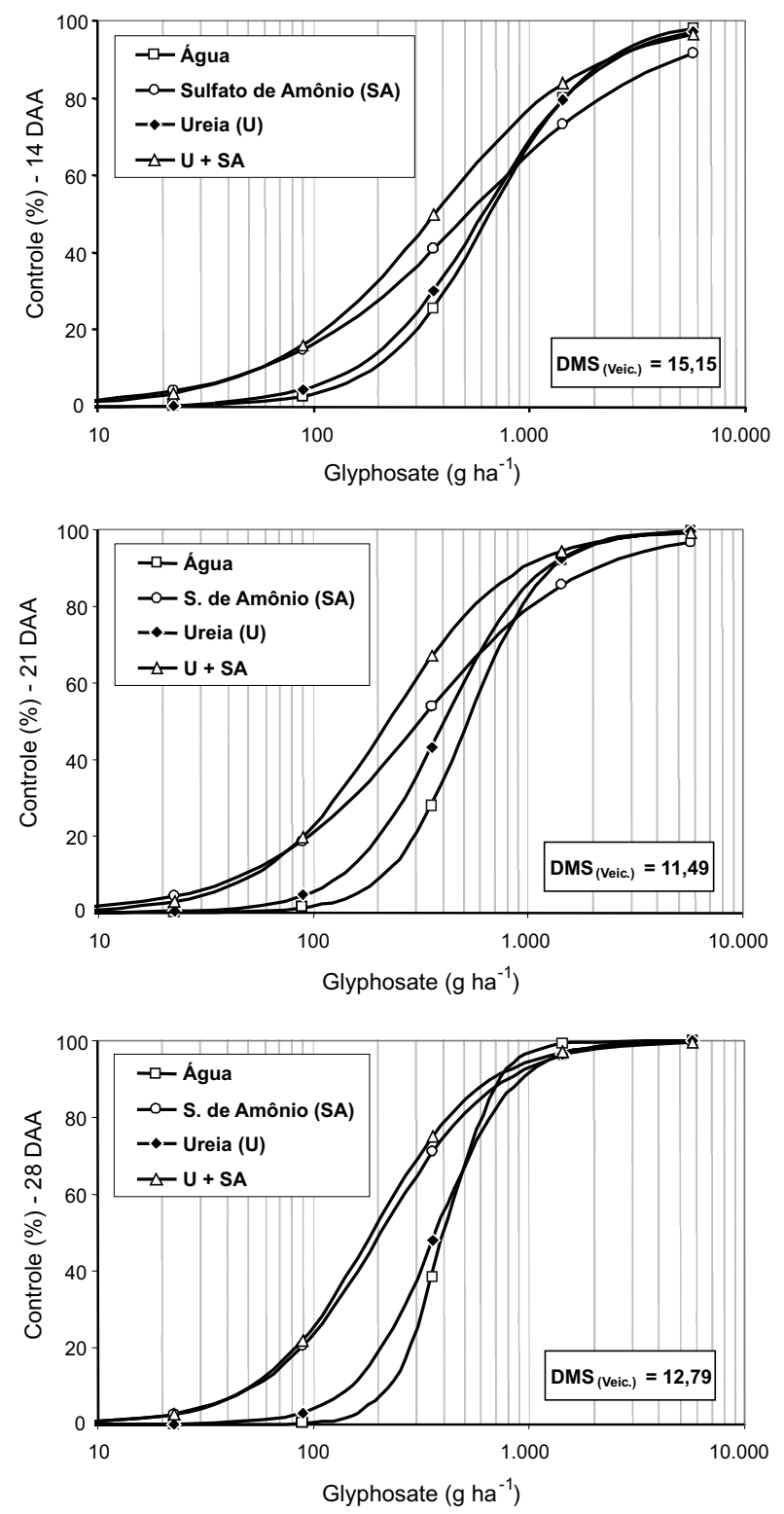

Figura 1 - Controle percentual do capim-amargoso (D. insularis) quando submetido a diferentes doses do herbicida glyphosate, avaliado aos 14, 21 e 28 dias após aplicação (DAA), utilizando como veículo água pura, solução de sulfato de amônio $\left(15 \mathrm{~g} \mathrm{~L}^{-1}\right)$, solução de ureia $\left(5 \mathrm{~g} \mathrm{~L}^{-1}\right) \mathrm{e}$ solução de ureia + sulfato de amônio $\left(2,5+7,5 \mathrm{~g} \mathrm{~L}^{-1}\right)$. Piracicaba-SP, 2008 dos tratamentos, em que menos massa foi constatada nas parcelas que receberam aplicações da maior dose de glyphosate ou da menor dose acrescida de U+SA. Não foram observadas diferenças entre os tratamentos herbicidas para a variável massa seca (Tabela 4). A ocorrência de diferenças no controle e na massa fresca está diretamente relacionada com a ação dessecante do herbicida glyphosate, diferencial em cada tratamento; essa diferença é atenuada após a exposição igualitária do material de todos os tratamentos ao processo de secagem em estufa.

A maior eficácia promovida pela aplicação de glyphosate com solução de U+SA, provavelmente, é consequência das características complementares que esses fertilizantes nitrogenados possuem quando em solução. Quanto ao sulfato de amônio, as principais atividades benéficas reconhecidas têm sido o antagonismo de cátions oriundos de água dura (Nalewaja \& Matysiak, 1993; Thelen et al., 1995; Gauvrit, 2003); a formação de sais glyphosate-amônio, que possuem maior facilidade de absorção (Nalewaja et al., 1992; Thelen et al., 1995); e a acidificação do apoplasto foliar, que facilita a absorção celular de herbicidas ácido fracos (Gronwald et al., 1993; Ruiter \& Meinen, 1996; Young et al., 2003).

O principal mecanismo de incremento de controle relatado para a ureia diz respeito à contribuição na penetração cuticular, devido à difusão facilitada dessa molécula, e ao rompimento de ligações éster, éter e diéter da cutina

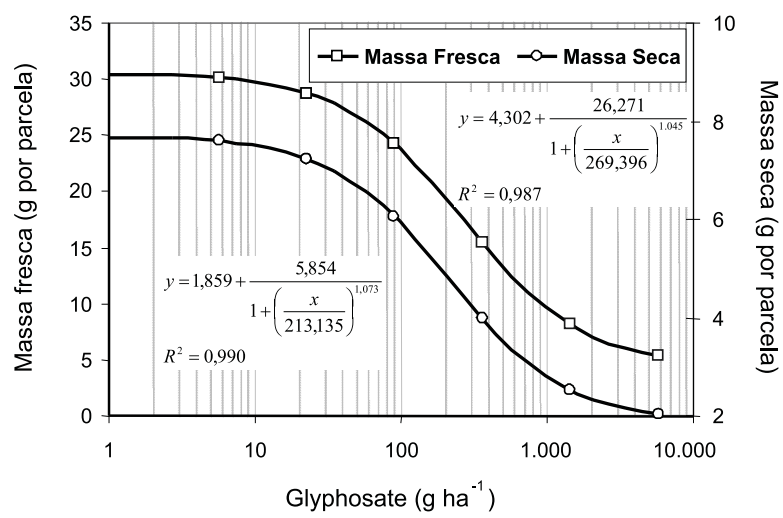

Figura 2 - Massa fresca e massa seca do capim-amargoso (D. insularis) quando submetido a diferentes doses do herbicida glyphosate, avaliadas aos 28 dias após aplicação. Piracicaba-SP, 2008. 
(Yamada et al., 1965; Durigan, 1992; Witte et al., 2002). Contudo, há evidências de que a penetração da ureia ocorre com a molécula intacta (Yamada et al., 1965). No citoplasma das células, em presença de urease, a ureia é rapidamente submetida à conversão por hidrólise, a qual origina duas moléculas de amônio e consome íons hidrogênio (Hinsvark et al., 1953; Chen \& Ching, 1988). A hidrólise da ureia contribui para elevação do $\mathrm{pH}$ citoplasmático, resultando, inclusive, na possibilidade da perda de nitrogênio na forma de amônia (Nielsen \& Schjoerring, 1998; Witte et al., 2002).

Nesse sentido, a caracterização precisa dos veículos de aplicação tem grande importância na compreensão dos resultados de controle e massa. $\mathrm{O}$ pH e o teor de amônio dos quatro veículos utilizados para aplicação de glyphosate no primeiro experimento estão apresentados na Tabela 5. Notadamente, os valores obtidos para a água comum estão em concordância com a análise completa, realizada previamente (Tabela 1). O pH da água comum, da ordem de 7,3 , foi reduzido em decorrência da dissolução de sulfato de amônio, tanto isolado quanto combinado com a ureia, com estabilização em 6,7. Resultados semelhantes também foram observados por McCormick (1990), que relatou redução média de 0,57 unidade de $\mathrm{pH}$ em amostras de água após adição de sulfato de amônio, sem a presença de herbicidas. Por outro lado, a dissolução de somente ureia em água promoveu a elevação do $\mathrm{pH}$ para 8,0 (Tabela 5).

Tabela 4 - Controle percentual, massa fresca e massa seca médias do capim-amargoso (D. insularis), após aplicação de seis tratamentos herbicidas com adição de diferentes fontes nitrogenadas (FN), avaliados aos 14, 21 e 28 dias após aplicação (DAA) $)^{1 /}$, em condição de casa de vegetação. Piracicaba-SP, 2008

\begin{tabular}{|c|c|c|c|c|c|c|c|}
\hline \multirow{3}{*}{ Tratamento } & \multicolumn{2}{|c|}{ Dose } & \multirow{2}{*}{\multicolumn{3}{|c|}{$\begin{array}{c}\text { Controle percentual } \\
\text { (dias após aplicação) }\end{array}$}} & \multirow{2}{*}{\multicolumn{2}{|c|}{$\frac{\text { Massa }}{\text { (g por parcela) }}$}} \\
\hline & \multirow{2}{*}{$\begin{array}{c}\text { Herbicida } \\
\left(\mathrm{g} \mathrm{ha}^{-1}\right)\end{array}$} & \multirow{2}{*}{$\begin{array}{c}\mathrm{FN}^{4 /} \\
\left(\mathrm{g} \mathrm{L}^{-1}\right) \\
\end{array}$} & & & & & \\
\hline & & & 14 & 21 & 28 & Fresca & Seca \\
\hline Testemunha sem aplicação & -- & -- & $0,0 \mathrm{C}$ & $0,0 \mathrm{D}$ & $0,0 \mathrm{D}$ & $26,78 \mathrm{C}$ & $5,63 \mathrm{~B}$ \\
\hline Glyphosate $^{2 /}$ & 360 & -- & $30,0 \mathrm{~B}$ & $23,3 \mathrm{C}$ & $21,7 \mathrm{C}$ & $14,41 \mathrm{~B}$ & $2,92 \mathrm{~A}$ \\
\hline Glyphosate $+\mathrm{SA}^{3 /}$ & 360 & 15,0 & $33,7 \mathrm{~B}$ & $47,5 \mathrm{~B}$ & $48,8 \mathrm{~B}$ & $11,70 \mathrm{~B}$ & $2,61 \mathrm{~A}$ \\
\hline Glyphosate + Ureia (U) & 360 & 5,0 & $30,3 \mathrm{~B}$ & $33,3 \mathrm{C}$ & $27,2 \mathrm{C}$ & $14,03 \mathrm{~B}$ & $2,85 \mathrm{~A}$ \\
\hline Glyphosate + U + SA & 360 & $2,5+7,5$ & $44,5 \mathrm{~A}$ & $66,7 \mathrm{~A}$ & $68,7 \mathrm{~A}$ & $7,50 \mathrm{~A}$ & $1,95 \mathrm{~A}$ \\
\hline Glyphosate & 720 & -- & $42,8 \mathrm{~A}$ & $65,0 \mathrm{~A}$ & $75,8 \mathrm{~A}$ & $8,84 \mathrm{~A}$ & $2,22 \mathrm{~A}$ \\
\hline \multicolumn{3}{|c|}{$\mathrm{F}_{\text {(trat) }}$} & $53,47 * *$ & $53,83 * *$ & $47,92 * *$ & $13,19 * *$ & $14,19 * *$ \\
\hline \multicolumn{3}{|c|}{$\mathrm{CV}(\%)$} & 17,79 & 21,86 & 25,66 & 33,50 & 28,53 \\
\hline
\end{tabular}

${ }^{1 /}$ Médias seguidas por letras iguais, nas colunas, não diferem entre si pelo teste de agrupamento de médias de Scott-Knott a 5\% de probabilidade;

${ }^{2 /}$ Roundup Original ${ }^{\circledast}, 360 \mathrm{~g} \mathrm{~L}^{-1} ;{ }^{3 /}$ Sulfato de amônio; ${ }^{4 /}$ Fontes nitrogenadas; **significativo a $1 \%$ de probabilidade pelo teste $\mathrm{F}$.

Tabela 5 - Quantificação do $\mathrm{pH}$ e dos teores de amônio nos quatro veículos utilizados para aplicação das diferentes doses de glyphosate. Piracicaba-SP, 2008

\begin{tabular}{|l|c|c|}
\hline \multirow{2}{*}{ Veículo de aplicação } & \multicolumn{2}{|c|}{ Parâmetro químico ${ }^{1 /}$} \\
\cline { 2 - 3 } & $\mathrm{pH}$ & $\mathrm{N}^{1 /} \mathrm{NH}_{4}^{+}\left(\mathrm{mg} \mathrm{L}^{-1}\right)$ \\
\hline Água comum & 7,30 & 0,13 \\
\hline Solução de Sulfato de Amônio (SA) $-15 \mathrm{~g} \mathrm{~L}^{-1}$ & 6,70 & 3821,45 \\
\hline Solução de Ureia (U) $-5 \mathrm{~g} \mathrm{~L}^{-1}$ & 8,00 & 3,18 \\
\hline Solução de U $+\mathrm{SA}-2,5+7,5 \mathrm{~g} \mathrm{~L}^{-1}$ & 6,73 & 1585,20 \\
\hline
\end{tabular}

1/ A amostra de cada veículo foi analisada por duas vezes, o que não justifica a realização de análise estatística. 
Na solução de SA, de acordo com o cálculo molar, eram esperados $4.090 \mathrm{mg} \mathrm{L}^{-1}$ de amônio, o que não foi confirmado pela análise química, que indicou $3.821,45 \mathrm{mg} \mathrm{L}^{-1}$ (Tabela 5). Além de possiveis impurezas presentes no fertilizante, essa diferença de $6,5 \%$ se deve, provavelmente, à perda de nitrogênio por volatilização de amônia $\left(\mathrm{NH}_{3}\right)$, o que auxilia na explicação da redução no pH. Segundo Damin (2009), em solução aquosa, parte dos íons amônio é convertida em $\mathrm{NH}_{3}$ conforme a seguinte reação de equilíbrio:

$$
\mathrm{NH}_{4}^{+} \leftrightarrow \mathrm{NH}_{3}+\mathrm{H}^{+}
$$

Essa reação tem $\mathrm{pKa}=9,2$ (constante de equilíbrio), ou seja, neste $\mathrm{pH}$ metade das moléculas é encontrada na forma de amônio $\left(\mathrm{NH}_{4}^{+}\right)$ e metade na forma de amônia $\left(\mathrm{NH}_{3}\right)$. A acidificação da calda após dissolução de sulfato de amônio (Tabela 5) indica a liberação de íons hidrogênio e, proporcionalmente, de amônia, a qual, por seu caráter volátil, é perdida para a atmosfera (Witte et al., 2002).

A U, por sua vez, precisa ser convertida por hidrólise para formação de amônio. Nas plantas e no solo essa reação é mediada por ureases, de modo que a hidrólise espontânea é bastante reduzida. A reação completa de hidrólise da ureia $\left(\mathrm{CO}\left(\mathrm{NH}_{2}\right)_{2}\right)$ pode ser representada pela seguinte equação (Hinsvark et al., 1953; Witte et al., 2002):

$$
\mathrm{CO}\left(\mathrm{NH}_{2}\right)_{2}+\mathrm{H}_{2} \mathrm{O}+2 \mathrm{H}^{+} \stackrel{\text { urease }}{\leftrightarrow} 2 \mathrm{NH}_{4}^{+}+\mathrm{CO}_{2}
$$

Notadamente, para que a reação ocorra há consumo de íons hidrogênio, o que resulta na elevação do pH da solução, conforme observado na Tabela 5. Também no caso da solução U+SA, o teor de amônio mensurado (1.585,20 $\left.\mathrm{mg} \mathrm{L}^{-1}\right)$ não é a metade da solução isolada de SA $\left(3.821,45 \mathrm{mg} \mathrm{L}^{-1}\right)$. Nesse caso, sugere-se que a hidrólise espontânea da ureia exerceu função de dreno de $\mathrm{H}^{+}$, provocando maior dissociação de moléculas de amônio para estabilização do $\mathrm{pH}$, da ordem de 6,7. Nessas condições, possivelmente houve maior perda de nitrogênio para a atmosfera sob a forma de amônia (Tabela 5).

Em termos práticos, a partir dos dados da Tabela 5 , pode-se supor que há pequena perda de amônia para a atmosfera quando é utilizada solução de SA e que essas perdas são maiores para solução de U+SA. Ainda, a maior parte da ureia se mantém preservada em solução, de modo que, quando considerado esse veículo, o que alcança as folhas não é amônio. Por outro lado, a alteração do $\mathrm{pH}$ da calda provocada por esses fertilizantes nitrogenados não tem grande importância após a dissolução do glyphosate. Na literatura científica, tem-se observado significativo efeito tamponante desse herbicida, sobretudo quando da aplicação de doses superiores a $10 \mathrm{~mL} \mathrm{~L}^{-1}$ de produto comercial (Nalewaja \& Matysiak, 1991; Carvalho et al., 2009a).

Assim, a adoção da solução de sulfato de amônio + ureia como veículo de aplicação do herbicida glyphosate elevou a eficácia do produto sobre o capim-amargoso (Digitaria insularis). Essas observações sugerem efeito complementar dos fertilizantes nitrogenados. Para isso, supõe-se que a ureia tenha contribuído para a melhor penetração foliar, enquanto o sulfato de amônio facilitou a absorção celular do herbicida. Contudo, em outros casos, essas alterações podem não ser observadas. Resultados satisfatórios dessa mistura são esperados para as espécies daninhas em que há impedimento à penetração cuticular e absorção celular. No entanto, para espécies em que a penetração cuticular não é impedimento, supõe-se que aplicações de glyphosate somente com sulfato de amônio sejam mais eficazes. Novos trabalhos devem ser desenvolvidos, visando avaliar a eficácia da combinação de glyphosate com U+SA sobre outras espécies de plantas daninhas, em condição de campo, bem como alterações na proporção de U e SA na mistura.

\section{AGRADECIMENTOS}

Ao Conselho Nacional de Desenvolvimento Científico e Tecnológico (CNPq), pela concessão de bolsas.

\section{LITERATURA CITADA}

BREMMER, J. M.; KEEENEY, D. R. Determination and isotope ratio analysis of different forms of nitrogen in soil. III. Exchangeable ammonium, nitrate and nitrite by steamdistillation methods. Soil Sci. Soc. Am. Proc., v. 30, n. 5, p. $577-582,1966$.

CARVALHO, S. J. P. et al. Curvas de dose-resposta para avaliação do controle de fluxos de emergência de plantas daninhas pelo herbicida imazapic. Planta Daninha, v. 23, n. 3, p. 535-542, 2005. 
CARVALHO, S. J. P. et al. Glifosato aplicado com diferentes concentrações de ureia ou sulfato de amônio para dessecação de plantas daninhas. Pesq. Agropec. Bras., v. 43, n. 11, p. $1501-1508,2008$.

CARVALHO, S. J. P. et al. Eficácia e pH de caldas de glifosato após a adição de fertilizantes nitrogenados e utilização de pulverizador pressurizado por $\mathrm{CO}_{2}$. Pesq. Agropec. Bras., v. 44, n. 6, p. 569-575, 2009a.

CARVALHO, S. J. P. et al. Dessecação de plantas daninhas com glyphosate em mistura com ureia ou sulfato de amônio. Planta Daninha, v. 27, n. 2, p. 353-361, 2009 b.

CHEN, Y.; CHING, T. M. Induction of barley leaf urease. Plant Physiol., v. 86, n. 3, p. 941-945, 1988.

CHRISTOFFOLETI, P. J. Curvas de dose-resposta de biótipos resistente e suscetível de Bidens pilosa L. aos herbicidas inibidores da ALS. Sci. Agric., v. 59, n. 3, p. 513-519, 2002.

CHRISTOFFOLETI, P. J.; LÓPEZ-OVEJERO, R. F. Definições e situação da resistência de plantas daninhas aos herbicidas no Brasil e no mundo. In: CHRISTOFFOLETI, P. J. (Coord.) Aspectos de resistência de plantas daninhas a herbicidas. 2.ed. Campinas: Associação Brasileira de Ação a Resistência de Plantas aos Herbicidas (HRAC-BR), 2004. p. 3-22.

DAMIN, V. Transformações do nitrogênio no sistema solo-planta após aplicação de herbicidas. 2009. 130 f. Tese (Doutorado em Agronomia) - Escola Superior de Agricultura "Luiz de Queiroz", Piracicaba, 2009.

DONALD, W. W. Established foxtail barley, Hordeum jubatum, control with glyphosate plus ammonium sulfate. Weed Technol., v. 2, n. 3, p. 364-368, 1988.

DURIGAN, J. C. Efeito de adjuvantes na calda e no estádio de desenvolvimento das plantas, no controle do capimcolonião (Panicum maximum) com glyphosate.

Planta Daninha, v. 10, n. 1/2, p. 39-44, 1992.

FRANSON, M. A. H. Standard methods for the examination of water and wastewater. 19.ed. Washington: American Public Health Association, 1995. 1074 p.

FREIRE, M. F. et al. Nutrição foliar: princípios e recomendações. Inf. Agropec., v. 7, n. 81, p. 54-62, 1981.

GAUVRIT, C. Glyphosate response to calcium, ethoxylated amine surfactant, and ammonium sulfate. Weed Technol., v. 17, n. 4 , p. $799-804,2003$

GEIGER, D. R.; FUCHS, M. A. Inhibitors of aromatic amino acid biosynthesis (glyphosate). In: BÖGER, P.; WAKABAYASHI, K; HIRAI, K. (Eds.) Herbicide classes in development. Berlin: Springer-Verlag, 2002 . p. 59-85.
GRONWALD, J. W. et al. Effect of ammonium sulfate on absorption of imazethapyr by quackgrass (Elytrigia repens) and maize (Zea mays) cell suspension cultures. Weed Sci., v. 41, n. 3, p. 325-334, 1993.

HISNVARK, O. N.; WITTWER, S. H.; TUKEY, H. B. The metabolism of foliar-applied urea. I. Relative rates of $\mathrm{C}^{14} \mathrm{O}_{2}$ production by certain vegetable plants treated with labeled urea. Plant Physiol., v. 28, n. 1, p. 70-76, 1953.

JORDAN, D. L. et al. Influence of application variables on efficacy of glyphosate. Weed Technol., v. 11, n. 2, p. 354362, 1997.

MACHADO, A. F. L. et al. Caracterização anatômica de folha, colmo e rizoma de Digitaria insularis.

Planta Daninha, v. 26, n. 1, p. 1-8, 2008.

McCORMICK, R. W. Effects of $\mathrm{CO}_{2}, \mathrm{~N}_{2}$, air and nitrogen salts on spray solution $\mathrm{pH}$. Weed Technol., v. 4, n. 4, p. $910-912,1990$.

MONQUERO, P. A. et al. Absorção, translocação e metabolismo do glyphosate por plantas tolerantes e suscetíveis a este herbicida. Planta Daninha, v. 22, n. 3, p. 445-451, 2004.

NALEWAJA, J. D.; MATYSIAK, R. Optimizing adjuvants to overcome glyphosate antagonistic salts. Weed Technol., v. 7, n. 2, p. 337-342, 1993.

NALEWAJA, J. D.; MATYSIAK, R. Salt antagonism of glyphosate. Weed Sci., v. 39, n. 4, p. 622-628, 1991

NALEWAJA, J. D.; MATYSIAK, R.; FREEMAN, T. P. Spray droplet residual of glyphosate in various carriers. Weed Sci., v. 40, n. 4, p. 576-589, 1992.

NIELSEN, K. H.; SCHJOERRING, J. K. Regulation of apoplastic $\mathrm{NH}_{4}^{+}$concentration in leaves of oilseed rape. Plant Physiol., v. 118, n. 4, p. 1361-1368, 1998.

PRATT, D.; KELLS, J. J.; PENNER, D. Substitutes for ammonium sulfate as additives with glyphosate and glufosinate. Weed Technol., v. 17, n. 3, p. 576-581, 2003.

RODRIGUES, B. N.; ALMEIDA, F. S. Guia de herbicidas. 5.ed. Londrina: 2005. 592 p.

RUITER, H. D.; MEINEN, E. Adjuvant-increased glyphosate uptake by protoplasts isolated from quackgrass Elytrigia repens (L.) Nevski. Weed Sci., v. 44, n. 1, p. 38-45, 1996.

SATCHIVI, N. M. et al. Absorption and translocation of glyphosate isopropylamine and trimethysulfonium salts in Abutilon theophrasti and Setaria faberi. Weed Sci., v. 48, n. 6, p. $675-679,2000$. 
SCOTT, A. J.; KNOTT, M. A. Cluster analysis method for grouping means in the analysis of variance. Biometrics, v. 30, n. 2 , p. $507-512,1974$.

SEEFELDT, S. S.; JENSEN, S. E.; FUERST, E. P. Log-logistic analysis of herbicide dose-response relationship.

Weed Technol., v. 9, n. 2, p. 218-227, 1995.

SILVA, J. F. et al. Herbicidas: absorção, translocação, metabolismo, formulação e misturas. In: SILVA, A. A.; SILVA, J. F. (Eds.). Tópicos em manejo de plantas daninhas. Viçosa, MG: Universidade Federal de Viçosa, 2007. p. 149-188.

STREIBIG, J. C. Herbicide bioassay. Weed Res., v. 28, n. 6, p. 479-484, 1988.

THELEN, K. D.; JACKSON, E. P.; PENNER, D. The basis for the hard-water antagonism of glyphosate activity. Weed Sci., v. 43, n. 4, p. 541-548, 1995
TIMOSSI, P. C.; DURIGAN, J. C.; LEITE, G. J. Eficácia de glyphosate em plantas de cobertura. Planta Daninha, v. 24, n. 3, p. 475-480, 2006.

WITTE, C. P. et al. Leaf urea metabolism in potato. Urease activity profile and patterns of recovery and distribution of ${ }^{15} \mathrm{~N}$ after foliar urea application in wild-type and ureaseantisense transgenics. Plant Physiol., v. 128, n. 3, p. $1129-1136,2002$

YAMADA, Y; WITTWER, S. H.; BUKOVAC, M. J. Penetration of organic compounds through isolated cuticular membranes with special reference to $\mathrm{C}^{14}$ urea. Plant Physiol., v. 40, n. 1 , p. $170-175,1965$

YOUNG, B. G. et al. Glyphosate translocation in common lambsquarters (Chenopodium album) and velvetleaf (Abutilon theophrasti) in response to ammonium sulfate. Weed Sci., v. 51, n. 2 , p. $151-156,2003$ 